\title{
The Influence of a Hand Preference for Acquiring Objects on the Development of a Hand Preference for Unimanual Manipulation from 6 to 14 Months
}

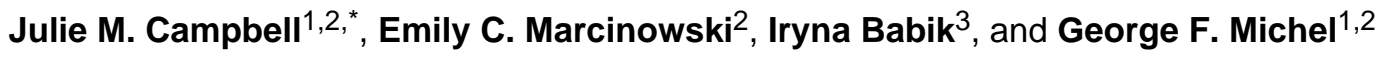 \\ ${ }^{1}$ Center for Developmental Science, University of North Carolina at Chapel Hill, Chapel Hill, NC, \\ United States. \\ ${ }^{2}$ Department of Psychology, University of North Carolina at Greensboro, Greensboro, NC, United \\ States. \\ ${ }^{3}$ Department of Physical Therapy, University of Delaware, Newark, DE, United States.
}

\begin{abstract}
Development of hand preferences for unimanual manipulation of objects was explored in 90 infants (57 males) tested monthly from 6 to 14 months. From a larger sample of 380 infants, 30 infants with a consistent left hand preference for acquiring objects were matched for sex and development of locomotion skills with 30 infants with a consistent right hand preference for acquisition and 30 with no preference. Although frequency of unimanual manipulations increased during 6-14 month period, infants with a hand preference for acquisition did more object manipulations than those without a preference for acquisition. Multilevel modeling of unimanual manipulation trajectories for the three hand-preference groups revealed that hand preferences for unimanual manipulation become more distinctive with age, and the preference is predicted by the hand preference for object acquisition. Infants with a right and left hand preference for object acquisition develop a right and left (respectively) hand preference for unimanual manipulation. However, the majority of infants at each month do not exhibit hand preferences for unimanual manipulation that are unlikely to occur by chance, even by 14 months. The results are consistent with a cascading theory of handedness development in which early preferences (i.e., for acquisition) are transferred to later developing preferences (i.e., for unimanual manipulation).
\end{abstract}

\section{Keywords}

infant handedness; unimanual manipulation; lateralization; longitudinal

\footnotetext{
(C) 2015 Published by Elsevier Inc.

*Corresponding Author: University of North Carolina Greensboro, Department of Psychology, 296 Eberhart Building, Greensboro, NC, 27402, Phone: (336) 334-4046, Fax: (336) 334-5066, jmcampb2@uncg.edu.

Publisher's Disclaimer: This is a PDF file of an unedited manuscript that has been accepted for publication. As a service to our customers we are providing this early version of the manuscript. The manuscript will undergo copyediting, typesetting, and review of the resulting proof before it is published in its final citable form. Please note that during the production process errors may be discovered which could affect the content, and all legal disclaimers that apply to the journal pertain.
} 
When engaging with objects, infants use their arms and hands in a variety of ways: swiping, grasping, unimanual manipulation (e.g., banging, shaking, hitting, throwing), roledifferentiated bimanual manipulation (RDBM - one hand supports the active manipulation of the object by the other hand). Each of these uses contributes to a variety of accomplishments with the objects (e.g., tool-use, problem solving). Uzgiris and Hunt (1975) proposed that unimanual manipulation of objects develops from earlier skills such as reaching for and grasping (acquiring) objects. Therefore, a hand preference for acquiring objects could transfer into a preference for manipulating them. The primary goal of this study is to determine whether an infant's earlier appearing hand preference when reaching for and grasping (acquiring) objects predicts the later development of a hand preference for manipulating them with one hand.

For infants, hand preferences can be identified only by observing their differential use in particular situations. Under these conditions, the difference in use between the hands is distributed continuously across infants with a majority exhibiting more frequent right- than left- hand use (e.g., Ferre, Babik, \& Michel 2010, for acquiring objects) similar to adult proficiency differences (Annett, 2002). If handedness is a continuously distributed characteristic, then assigning categories of "right" and "left" can only refer to relative membership in a particular population or sample rather than as referring to something intrinsic to the individual.

Michel (2002) proposed that during development, infant hand preferences are transferred across prehensile manual skills (beginning with visually-elicited swiping at objects, extending to visually-guided acquisition of objects, to unimanual manipulation, and eventually to RDBM - role-differentiated bimanual manipulation). As a result of such transfer effects during development, the individual eventually has a relatively consistent preference across many manual skills which can form the basis of the trait-like character of handedness. The current study is designed to assess this proposal by examining whether preferences for acquiring objects will concatenate into preferences for manipulating them.

Hinojosa, Sheu, and Michel (2003) provided some support for this concatenation proposal when they assessed unimanual manipulation at 7 and 11 months for infants with different hand preferences for acquiring objects. At 7 months, few infants had a hand preference for manipulating objects (Hinojosa et al., 2003). However, by 11 months, infants who manifested right hand preference for acquiring objects at 7, 9, and 11 months exhibited a right hand preference for unimanual manipulation. Infants with a left hand preference for acquisition at 7,9, and 11 months had a left hand preference for unimanual manipulation. The infants without a hand preference for acquiring objects (the differences between their hands in frequency of use were likely to occur by chance for each month) did not exhibit a hand preference for manipulation at either 7 or 11 months. Thus, there appeared to be a predictive relation between a hand preference for acquiring objects and the subsequent development of a hand preference for unimanual manipulation.

Unfortunately, the study by Hinojosa and colleagues had two deficiencies: 1) a rather small sample of 25 infants ( 10 with a right preference and 8 with a left preference for acquiring objects) was tested and only at 7, 9, and 11 months of age; 2) for the unimanual assessment 
task, the infant was allowed to acquire spontaneously each object. Thus, those infants with a hand preference for acquiring objects likely initiated unimanual manipulation with their preferred hand for acquiring objects. This could bias any association of the unimanual manipulation hand preference with the preference for acquisition. However, the absence of a relation between the two preferences at seven months likely indicates the independence of the hand preferences for these two manual actions. In the current study, we avoid this second deficiency by placing pairs of identical objects simultaneously in each of the infant's hands, thereby promoting manual symmetry for object acquisition. Thereafter, any differences between the hands in manipulation frequency (e.g., shake, bang) are not a consequence of a preference for acquiring the object. Moreover, we assess the hand preference of 90 infants monthly (nine times) from 6 to 14 months of age.

Previous research has used the frequency of manual actions as a marker for evaluating the development of an infant's engagement in various unimanual manipulations (Hinojosa et al., 2003; Kimmerle, Mick, \& Michel, 1995; Kimmerle, Ferre, Kotwica, \& Michel, 2010). Therefore, we use "unimanual manipulation" to refer to the number of unimanual actions that are performed on an object. Hinojosa et al. (2003) reported no significant change in frequency of unimanual manipulation actions between ages 7 and 11 months for 25 infants. Similar results were reported by Kimmerle et al. (2010), for 14 infants tested bimonthly during the 7 to 13 month age period when playing with 6 toys. Although some unimanual actions, such as manipulation of the infant's body parts (other hand, feet, lips, ears) and clothing, appear very early after birth, these actions reflect more primary and secondary circular reactions (Baldwin, 1894; Piaget, 1952) than controlled actions on objects.

According to Ramsay (1980), the earliest instance of unimanual manipulation of objects is at 5 months of age. He observed infants' unimanual actions with four toys in a cross-sectional design at 5, 7 and 9 months $(n=16$ at each age) and reported that the total number of unimanual actions increased significantly between 5 and 7 months of age but not thereafter.

Thus, several studies observed no change in frequency of unimanual manipulation actions from seven months on (Hinojosa et al., 2003; Kimmerle et al., 2010; Kimmerle et al., 1995; Ramsay, 1980). However, a hand preference for acquiring objects seems to appear as early as six months of age (e.g., Ferre et al., 2010; Michel, Babik, Sheu, \& Campbell, 2014). Therefore, if a unimanual preference is simply another manifestation of the same underlying mechanism controlling the acquisition preference, we might expect that a unimanual hand preference would appear soon after six months of age. In contrast, if unimanual manipulation preferences are transferred from hand preferences for acquisition, we would predict that unimanual preferences would develop much later (about 5 months) in infancy.

The current study assesses unimanual actions monthly during the 6 to 14 month age period for a group of 60 infants whose hand preference for acquiring objects remained consistent during that age period (30 with a right hand preference and 30 with a left preference) and a group of 30 infants who did not exhibit a consistent hand preference for acquiring objects during this age period. We examine, monthly, the development of differences between the hands in their frequency of performance of eight unimanual manipulation actions. We identify hand-use preference categories in two ways. First, at each month of age, the relative frequency of left and right hand-use for that assessment (which distributes continuously 
across infants within an age) is categorized into "right" or "left" hand preference according to the significance of the difference in frequency of use between the hands $(a<.01)$. Differences that do not differ from chance are assigned to a "no preference" category. Second, infants are categorized into "right", "left", and "no preference" according to the latent classes revealed via the analysis of the trajectories of their relative hand-use across the nine monthly assessments from 6 to 14 months of age.

We predict that hand preferences for unimanual manipulation will become more distinctive with age. We also predict a transfer of the preference from acquisition to unimanual manipulation. Thus, those infants with a right hand preference for object acquisition will develop a right hand preference for unimanual manipulation. Similarly, those with a left hand preference for object acquisition will develop a left hand preference for unimanual manipulation. Infants without a preference for acquiring objects will be unlikely to exhibit a preference for unimanual manipulation and likely represent the early development of those adults who exhibit rather small differences between their hands in manual proficiency. Thus, by 14 months, hand preferences for unimanual manipulation are predicted to become consistent with the hand preferences for object acquisition.

\section{Method}

\subsection{Participants}

The sample of 90 infants ( 57 males, 33 females) used for this study is a subsample of 380 infants tested in the Infant Development Center at the University of North Carolina Greensboro. Enrollment of participants, informed consent, data collection and storage were completed in compliance with IRB regulations for the protection of human subjects. At each monthly visit, parents received a \$10 gift card. All infants had a normal gestation period and birth weight, and came from uncomplicated single births. The current sample is ethnically diverse: $71 \%$ Caucasian, 22\% African American, 5\% of Hispanic or Latino, and 2\% of mixed ethnicity. All subjects were tested monthly, within $+/-7$ days from infants' monthly birthdays, from 6 to 14 months (total 9 visits) on object acquisition and unimanual manipulation. Infants' mean age was 6.13 months ( $S D=0.15$ months) at the beginning of the study, and 14.25 months ( $S D=0.16$ months) at the end of the study. In the sample of 380 infants, 45 exhibited a consistent developmental trajectory with a left hand preference for acquiring objects during the age period of 6 to 14 months. Of these 45, 30 infants (19 males, 11 females) were randomly selected for this study and then matched for sex and the level of postural control and locomotion (onset of sitting, crawling, and walking) using the Touwen's (1976) scales with 30 infants with a consistent right hand-preference trajectory for acquisition and 30 infants without a distinct hand preference. The trajectories for hand preferences for acquiring objects were identified from the latent classes revealed by the Group Based Trajectory Modeling (GBTM, Nagin, 2005) technique applied for 380 infants.

\subsection{Procedure}

2.2.1 Object Acquisition-Object acquisition was defined as an action of grasping and maneuvering an object such as lifting it from the surface of the table. Hand preference for object acquisition was evaluated monthly from 6 to 14 months. Infants' manual activity 
during play with 34 infant toys was recorded using two synchronized cameras which provided both an overhead and a side view of the infant's hands. While infants sat on their parents' laps, parents were asked to stabilize the infant's waist to maintain a steady posture during play. Once the infant was seated at the table, a research assistant would present the items on the table directly in front of the infant. Each infant received a total of 32 presentations as either one toy on the table (17), one toy suspended in the air (5), a pair of identical toys on the table (7) or a pair of identical toys suspended in the air (3). Identical pairs were presented in line with the infant's shoulders, and single toys were presented to the infant's midline. The entire object acquisition procedure lasted 20-25 minutes. Infants were allowed to pick up the toys and explore the objects for up to 25 seconds before the research assistant removed the item and presented the next item.

Acquisition hand preference was coded in the Observer ${ }^{\circledR}$ XT (Noldus Information Technology, Wageningen, Netherlands) which permitted a frame-by-frame account of the hand used for an object acquisition. The hand initially used to acquire each toy was coded for all toys at each visit. Twenty percent of all coded videos were re-coded by another coder for inter-rater reliability (Cohen's Kappa $M=0.91, M d n=0.91$, range $=0.82$ to 0.99 ). Another $20 \%$ of the videos were re-coded for intra-rater reliability (Cohen's Kappa $M=$ $0.94, M d n=0.94$, range $=0.88$ to 0.99 ). Coders were unaware of infants' hand preference.

To analyze developmental trajectories of hand preference for object acquisition, the infant's monthly hand preferences for object acquisition were converted into Handedness Indexscores: $\mathrm{HI}=(\mathrm{R}-\mathrm{L}) /(\mathrm{R}+\mathrm{L})^{1 / 2}$, where $\mathrm{R}$ and $\mathrm{L}$ correspond to the total number of acquisitions performed by the right and the left hand. Next the GBTM (Nagin, 2005) and the SAS TRAJ procedure (Jones, Nagin, \& Roeder, 2001) were used on hand preference HI-scores to derive hand-preference latent classes from 380 infants' monthly (from 6 to 14 months) assessments (Michel et al. 2014). GBTM is a statistical method that permits identification of distinct patterns in the distribution of a sample's trajectories (Nagin, 2005) and is particularly useful in identifying heterogeneous subpopulations. The model assumed that there are unobserved latent groups in the population of infants and that these latent groups have distinct developmental trajectories of hand-use preference. The SAS TRAJ procedure (Jones et al., 2001) permitted fitting a series of mixture models to the data. The Bayesian information criterion (BIC) was used to identify the number of groups in the model (Schwarz, 1978). Specifically, $2 \Delta \mathrm{BIC}$, twice the difference between the BIC for the full model (larger number of groups) and that for the reduced model (smaller number of groups), is interpreted as the degree of evidence for the full model. This interpretation is justified because $2 \Delta \mathrm{BIC}$ is approximately $2 \ln B_{10}$, where $B_{10}$ is the Bayes factor (Kass \& Raftery, 1995). A value of $2 \ln B_{10}$ greater than 10 is interpreted as very strong evidence against the reduced model, which can be replaced in favor of the more complicated model (Kass \& Wasserman, 1995). The GBTM assigns infants to latent classes according to the highest associated classification probabilities.

Of a total of 45 infants, whose trajectory exhibited a significant and consistent left hand preference for acquiring objects, we randomly selected 30 infants and matched them (for sex and locomotor development) with 30 infants whose trajectory exhibited a significant right hand preference, and 30 infants without a hand preference throughout the 6 to 14 month age 
period. These 90 infants served as the subjects for this investigation of the relation of hand preference for acquiring objects to the hand preferences for unimanually manipulating objects.

2.2.2 Unimanual Manipulation-Unimanual manipulation is an action in which one hand has an active manipulating role on an object while the other hand does not support the object of the manipulation. Unimanual manipulation was studied longitudinally during play with a set of 17 pairs of identical infant toys (Figure 1). Items of each pair were placed simultaneously in the infant's hands. Ten unimanual manipulations (shaking, hitting, mouthing, rotating, scraping, clacking, picking up (only if an object was dropped by the infant), taking, refusing, and dropping) performed by each hand on each object were coded using the Noldus Observer ${ }^{\circledR}$ XT in real time until 6 manipulations had occurred. The hand used for each active manipulation was identified. "Shake" was coded for swinging of an object in a vertical orientation without a table contact; "hit" - several abrupt contacts of an object with the table; "in mouth" - placing an object in the mouth; "rotate" - turning the wrist in a circular motion; "scrape" - more than one sliding movement of an object across the table; "clack" - lateral movement of an object against another object in the opposite hand while that hand was inactive); "pick-up" - lifting a dropped object off of the table; "take" - removing an object from an inactive hand; "refuse" - refusing to accept an object from the presenter by pulling the hand away from the object; "drop" - termination of contact between the hand and an object. Repetitive actions were recorded only once unless another action intervened. For example, repetitive "shake" actions were recorded only once unless another action (e.g., "in mouth") occurred between bouts of shaking. Occasionally, an infant would drop a toy and proceed to engage the other toy with both hands. Such bimanual manipulations were not counted. Also, since there was a toy in each hand, both hands could be active simultaneously with a toy in each hand. These were recorded as "both" and were not included in the analyses because we were interested in only unimanual actions and not those that may have resulted from associated movements. For all further analyses, the actions of "refuse" and "drop" were not included because they likely represent some reluctance for using the hand and they remove the object from the hand or prevent the object from being manipulated.

Twenty percent of all coded videos were re-coded by another coder for inter-rater reliability (Cohen's Kappa $M=91, M d n=0.92$, range $=0.84$ to 0.96 ). Another $20 \%$ of the videos were re-coded for intra-rater reliability (Cohen's Kappa $M=0.93, M d n=0.94$, range $=0.86$ to 0.98). Coders were blind to infants' predicted hand preference. The number of coded rightand left-handed unimanual manipulations were converted into monthly HI-scores: $\mathrm{HI}=(\mathrm{R}$ $\mathrm{L}) /(\mathrm{R}+\mathrm{L})^{1 / 2}$ representing each infant's hand preference at each monthly visit. Multilevel analyses, using the Hierarchical Linear Modeling (HLM; Raudenbush, Bryk, Cheong, Congdon, \& du Toit, 2004), were then performed to explore developmental trajectories of the number of unimanual manipulations and hand preferences for unimanual manipulation according to the three groups (right, left, and no) defined by their hand preference for acquiring objects. The hand- use preference variable was coded as two dummy variables, "Left" and "NP", with Right being the reference group. 


\section{Results}

\subsection{Development of Hand Preference for Unimanual Manipulation}

We predicted that hand preferences for unimanual manipulation would become more distinctive with age. We also expected significant differences in hand preferences for unimanual manipulation among infants with different hand preference for acquiring objects. If an infant's HI-score was greater than 1.7 or less than -1.7 (this converts to a Z-score of transformation of binomial data in which when total right and left hand uses are greater than $24, a<0.01$, two-tailed, $\left(\mathrm{z}=((\mathrm{x}+/-0.5)-(0.5 \mathrm{~N})) /(0.25 \mathrm{~N})^{1 / 2}\right)$, Siegel, 1956), then this infant at this age was considered to have a significant hand preference. In the event that the total of right- and left-hand actions was less than 25 , the binomial probability was calculated. Infants with a right hand preference for object acquisition were predicted to develop a significant right hand preference for unimanual manipulation, whereas infants with a left hand preference for acquisition were predicted to develop a significant left hand preference for unimanual manipulation. Infants without a consistent hand preference for acquiring objects were predicted to exhibit no significant hand preference for manipulating objects during the six to 14 month period.

If we want to determine whether hand preference for acquisition is playing a role in the development of unimanual hand preference, then a hand preference for acquisition must be identified before the appearance of a hand preference for unimanual manipulation. Hinojosa et al. (2003) reported no unimanual hand preference before 11 months of age. Unimanual hand preference after 11 months of age can have been influenced by earlier hand preferences for acquisition if hand preference for acquisition is established and identified in earlier months.

Figure 2 shows that the mean hand preference scores (HI-scores) for acquiring objects were significantly different between the right and left preference infants at each age from 6 to 10 months. This difference starts out significant and increases across the months, which is consistent with their classification via the GBTM analysis. These results confirm that a hand use preference for acquisition has been established from 6 to 10 months of age. In order to support the concatenation theory, there would have to be no unimanual hand preference during this time. Note that the hand use scores for acquisition are significantly different between the two groups (t-test, Bonferroni corrected, $p<.01$ ) for each month, but only after 10 months are the hand use scores for unimanual manipulation significantly different between the groups, as marked by increasing use of the right hand by those infants with a right preference for acquiring objects.

In order to investigate the presence of a unimanual hand preference, we first conducted a multilevel analysis which revealed a significant quadratic trend of change in hand preference for unimanual manipulations (Table 1 and Figure 3). Note that linear, quadratic and cubic trends were analyzed in the model but only the significant trends are reported. Infants in each of the three hand preference groups for object acquisition (right, left, no preference) initially are not significantly different in their hand preference for unimanual manipulation (Tukey's HSD, $a>.10$ ). However, all infants increase their hand preference (HI scores) with age. Infants in the left hand-preference group for object acquisition increase the use of their 
left hand for unimanual manipulation with age and infants in the right hand-preference group for object acquisition increase their right hand preference for unimanual manipulation.

Only by 11 months of age, are the unimanual hand preference scores for infants in the right hand-preference group for acquisition significantly different from the unimanual preference scores of infants in the left hand-preference group for acquisition (Tukey's HSD, a $<.05$ ). Thereafter, infants with a right hand-preference for acquiring objects are always significantly different from left hand-preference infants in their hand preference for unimanual manipulation. By 13 and 14 months of age, the three acquisition preference groups (right, left, and no) are significantly different from one another in their hand preference for unimanual manipulation of objects (Tukey's HSD, $a<.05$ ) with infants having no acquisition preference exhibiting no preference for unimanual manipulation.

As predicted, a hand preference for unimanual manipulation becomes more distinctive with age in all infants and infant hand preferences for unimanual manipulation are predicted by their hand preferences for acquiring objects. Moreover, the infants in the left and right hand preference groups for acquiring objects are significantly different in their preference scores for acquisition at 6 months and on; whereas, they are only significantly different in their unimanual manipulation preference scores at 11 months - five months later. Only at 14 months were infants with no preference significantly different from both the left- and righthand preference groups. However, did each group differ significantly in their right and left unimanual hand use from an expected zero difference in right and left hand use from 12 month on?

Single sample t-tests were performed at 11,12,13, and 14 months of age to determine whether the mean $\mathrm{HI}$ for manipulation for each of the acquisition handedness groups differed from a population mean of zero. A conservative $\alpha=0.01$ was Bonferroni corrected for the four multiple comparisons for each group $(\alpha=0.0025)$. The Ts for those infants with a right-hand preference for acquisition were significant only for 13 and 14 months (11 month $\mathrm{T}=2.9, p=0.0035 ; 12$ month $\mathrm{T}=3.02, p=0.0026 ; 13$ month $\mathrm{T}=3.97, p<0.001$; 14 month $\mathrm{T}=5.86, p<0.0001$ ). Thus, only for the last two months of assessment was the right hand was used significantly more often than would be expected if the hands were used equivalently. None of the $\mathrm{T}$ values for those infants with a left-hand preference for acquisition were significant for any of the four months (11 month $\mathrm{T}=-0.46, p=0.326 ; 12$ month $\mathrm{T}=-2.45, p=0.01 ; 13$ month $\mathrm{T}=-1.25, p=0.11 ; 14$ month $\mathrm{T}=-0.72, p=0.24$ ). Thus, for all four months, the left hand was not used significantly more often than would be expected if the hands were used equivalently. Again, none of the $\mathrm{T}$ values were significant for those infants with no hand preference for acquisition (11 month $\mathrm{T}=0.68, p=0.25 ; 12$ month $\mathrm{T}=2.08, p=0.02 ; 13$ month $\mathrm{T}=1.71, p=0.098 ; 14$ month $\mathrm{T}=0.92, p=0.18$ ). Thus, with the exception of infants who preferred their right hand for acquiring objects, infants in each of the other two groups exhibited no significance difference between the use of their right and left hands.

To obtain more details about the development of lateralization for unimanual manipulation during 6 to 14 month period, we converted the relative hand scores (HI-scores) for each infant for each month into a categorical hand preference status for that infant at that month 
by using $\mathrm{HI}=+/-1.7$ (as described in footnote 1 ). Thus, if use of the right or the left hand for active unimanual manipulations was more frequent than would be expected by chance $(\mathrm{HI}=+/-1.7, \mathrm{a}<.01$, two-tailed), then the infant's hand preference status for manipulation was categorized as "right" or "left", respectively. If the difference in use of the two hands in active manipulations was not different from chance, then the infant's unimanual manipulation status was categorized as "no preference".

Table 2 shows the monthly unimanual hand preference status for infants relative to their hand preference status as defined by their latent class trajectory. The results reveal that at the ages 6 through 14 months, the majority of infants in each of the three groups with a hand preference status for object acquisition do not have a distinct hand preference for unimanual manipulation, even by 14 months of age. In addition, no significant differences among those with a right-preference, left-preference, and no preference for object acquisition are detected during six through 11 month period (6 months: $\chi^{2}(4, N=88)=0.920, p=.922 ; 7$ months: $\chi^{2}(4, N=89)=0.915, p=.922 ; 8$ months: $\chi^{2}(4, N=88)=4.879, p=.300 ; 9$ months: $\chi^{2}(4, N$ $=90)=2.081, p=.721 ; 10$ months: $\chi^{2}(4, N=88)=8.345, p=.080 ; 11$ months: $\chi^{2}(4, N=$ $90)=3.198, p=.525$ ). Note that occasionally (7 of 27 instances) an infant did not provide any usable data for assessing their unimanual manipulation preference.

After 11 months, statistically significant differences (all Bonferroni corrected) in the distribution of infants are identified for unimanual hand preferences for the three different hand preference groups for object acquisition (12 months: $\chi^{2}(4, N=90)=21.204, p<.0001$; 13 months: $\chi^{2}(4, N=90)=14.509, p=.006 ; 14$ months: $\left.\chi^{2}(4, N=90)=30.182, p<.0001\right)$. Note that at 12 months, the distribution of the three hand preference groups for unimanual manipulation across the three hand preference classes for object acquisition does not differ between right-handers and infants with no preference $\left(\chi^{2}(2, N=60)=2.783, p=.090\right)$, but differs significantly between right- and left-handed infants $\left(\chi^{2}(2, N=60)=12.444, p<\right.$. 002).

At 13 months, we also observed no significant difference between right-handers and no preference infants $\left(\chi^{2}(2, N=60)=4.044, p=.132\right)$ and a significant difference between right-handers and left-handers $\left(\chi^{2}(2, N=60)=12.121, p<.002\right)$. In contrast, at the age 14 months, not only do right-handers differ from left-handers $\left(\chi^{2}(2, N=60)=15.256, p<\right.$. $0001)$, but right- handers also become significantly different from infants with no hand preference $\left(\chi^{2}(2, N=60)=19.273, p<.0001\right)$. Again, these patterns suggest that the action of unimanual manipulation is developing during infancy from the stage of less lateralization towards increased lateralization and the direction of lateralized preference is predicted by their hand preference for acquisition.

We found no statistically significant change in hand preference distribution from age 7 months to 11 months (contrary to the results of Hinojosa et al., 2003): Right-preference

\footnotetext{
${ }^{1}$ The $\mathrm{HI}$ is derived from binomial data and the $\mathrm{z}$ transformation of the binomial is $\mathrm{z}=((\mathrm{x}+/-0.5)-(0.5 \mathrm{~N})) /(.25 \mathrm{~N})^{1 / 2}($ Siegel, $1956, \mathrm{p}$. 41). However, the formula $(\mathrm{R}-\mathrm{L}) /(\mathrm{R}+\mathrm{L})^{1 / 2}$ creates a pattern for frequencies greater than 25 actions that make the difference between right and left hand-use of 9 or larger for a total of 25 actions to have a $\mathrm{z}>2.00$ with a $\mathrm{p}<0.01$ ( 8 is not possible for this odd number). For a total of 30 actions, a difference of 10 or larger ( 9 is not possible) yields a $z>2.01$. Therefore, the formula (R-L)/(R+L) ${ }^{1 / 2}$ which results in $+/-1.7$ is equivalent to the $\mathrm{z}=2.0$ for any total actions greater than 25 . That is why we use $>+/-1.7$ as the decision criterion for assigning handedness.
} 
infants $\left(\chi^{2}(2, N=60)=2.00, p=.368\right)$, infants with no hand preference for object acquisition $\left(\chi^{2}(2, N=60)=0.591, p=.744\right)$, or left-preference infants $\left(\chi^{2}(2, N=59)=\right.$ $0.574, p=.750$ ). These results support the results that the hand preference for unimanual manipulation only begins to develop after the age of 11 months.

Finally, to test the consistency of the infant's unimanual hand use, four two-factor analyses of variance (one between and one within) were conducted on acquisition hand preference group (between) and four dependent variables (within) provided by the infant's HI score for unimanual manipulation: 1) The number of times an infant alternated between positive and negative HI scores across the nine months (more frequent alternations indicates greater inconsistency and we expected that infants without a preference for acquisition would be less consistent than either infants with a right or left preference for acquisition); 2) The number of positive $\mathrm{HI}$ scores observed for unimanual manipulation (more positive scores indicates more right hand use and we expected that infants who preferred to use their right hand for acquisition would have more positive HI scores than both infants who had no preference for acquisition and those with a left preference); 3) The number of alterations between significant HI scores (we predicted that infants without a hand preference for acquisition would show more alternations in their significant HI scores for unimanual manipulation than infants with either a right or left hand preference); 4) The number of significant HI scores for unimanual manipulation that an infant exhibited from 6 to 14 months (we expected that infants without a hand preference for acquisition would have fewer significant HI scores for unimanual manipulation than infants with either a right or left preference).

We found that infants with a right hand preference for acquiring objects exhibit the fewest alternations in $\mathrm{HI} \operatorname{sign}(M=1.3, S D=1.1)$ and no preference have the most $(M=3.76, S D=$ 1.4) with left in between $(M=2.3, S D=1.6)$. This frequency of alternations is significantly different for all three groups (Figure 4, Bonferroni corrected $\mathrm{p}=.003$ ) indicating that those infants with a right or left preference for acquisition are more consistent in their unimanual hand use than those without a preference $(F(2,87)=24.67, p<.0001)$.

As expected, infants with a right preference for acquisition also have the greatest number of positive HI scores $(M=8.0, S D=1.4)$ and those with a left preference for acquisition have significantly fewer positive (more negative) HI scores $(M=1.8, S D=1.1)$ than either those with a right preference or no preference $(F(2,87)=181.43, p<.0001)$. Also, infants with a right preference for acquisition have significantly more "significant" HI scores than those with either a left or no preference for acquisition $(F(2,87)=12.05, p<.0001)$. We observed that infants in the left preference groups have a less distinctive hand preference for unimanual manipulation than infants in the right preference group. Finally, as expected, infants in both the right and left acquisition preference groups exhibit significantly fewer alterations between significant $\mathrm{HI}$ scores $(\mathrm{HI}>|1.7|)$ than those without a preference $(F(2$, $87)=20.62, p<.0001)$. Thus, infants with either a right or left hand preference for acquisition exhibit more stable unimanual HI scores and these scores are consistent with their hand preference for acquisition. We conclude that the unimanual manipulation assessment identified relatively consistent hand-use that was consistent with the infant's acquisition preference. 
In order to account for the relative weakness of the unimanual HI scores of the infants with a left hand preference for acquisition, we examined mother's hand preference using the Briggs and Nebes (1975) modification of the Annett handedness questionnaire (Annett, 1972). Scores less than -9 or greater than +9 were categorized as left- and right-handed, respectively. These scores identified $86 \%$ right-handers and $9 \%$ left-handers in a reference group of 1599 adults ( $48 \%$ females). Amongst mothers of infants with a left hand preference, $85 \%$ were found to have a right hand preference, while only $8 \%$ were found to have a left preference. Amongst mothers of infants with a right hand preference, $78 \%$ were found to have a right hand preference, while only $9 \%$ were found to have a left preference. Previous research has reported that infant left-hand preference scores can be weakened by object play patterns with right-handed mothers (Michel, 1992).

\section{Discussion}

The goal of the current study was to examine the development of unimanual manipulation during 6 to 14 month period for infants with different hand preferences for acquiring objects. Starting at the age of 12 months, infants with a consistent hand preference (right or left) for acquiring objects perform more unimanual manipulations as compared to infants without a consistent hand preference for acquiring objects. Although it was proposed long ago (Cohen, 1966; Hildreth, 1949) that an early hand preference for acquiring objects would facilitate the later development of the more sophisticated manual skills involved in manipulating them, our results add to this relatively neglected issue of development (cf., Kotwica, Ferre, \& Michel, 2008).

Our observation of a significant quadratic trend in the development of the action of unimanual manipulation seems to contradict some of the previous research reporting no change in unimanual manipulation during infancy (Hinojosa et al., 2003; Kimmerle et al., 2010). However, in these previous studies, unimanual manipulation was assessed only at four ages (7, 9, 11, and 13 months) whereas the current study assessed unimanual manipulation at nine ages (6 through 14 months). The more frequent assessments permitted us to detect a quadratic trend of developmental change (cf., Ferre et al., 2010) with increases in unimanual manipulation achieving asymptote at a particular age. Thus, our results did confirm those of Hinojosa et al. (2003), since the mean number of unimanual manipulations in our study also did not significantly change between 7 and 11 months (36.2 vs. 37.4).

The results confirmed our predictions that hand preference for unimanual manipulation would become more distinctive with age and that a hand preference for acquiring objects would predict the hand preferred for unimanual manipulation. The results also confirmed our prediction that there would be a developmental delay in the expression of the preference for unimanual manipulation because the preference had to be transferred from acquiring objects to manipulating them. Infants with a right hand preference for acquiring objects (when compared to those with a left hand preference) initially are not significantly different in their hand use for unimanual manipulation. However, beginning at 11 months, those with a right hand preference for acquisition were significantly different from those with a left hand preference for acquisition in the hand used for unimanual manipulation. 
Although infants without a consistent hand preference for acquiring objects have a rightward developmental trajectory for unimanual manipulation hand preference, they remain relatively non-lateralized for manipulation, even at 14 months. These results are consistent with those reported by Hinojosa et al. (2003). Thus, a hand preference for acquisition predicts a subsequent hand preference for unimanual manipulation whereas no hand preference for acquisition was associated with no hand preference for manipulation. Nevertheless, most infants had not established reliable hand preferences even by 14 months of age. We propose that hand preferences for unimanual manipulation are still developing during the infant's second year but that development is being biased by the influence of the infant's hand preference for acquiring objects on the hand used for unimanual manipulation during the first year. Since hand preference status for object acquisition corresponds well with hand preference for unimanual manipulation, these results support the cascade theory of hand preference development (Michel et al., 2013).

Given that most infants do not exhibit significant hand preferences for unimanual manipulation even by 14 months, systematic longitudinal investigations of hand preferences must be conducted throughout the second year of infancy. There are a few such studies of this age period, but they typically begin at 18 months (Nelson, Campbell, \& Michel, 2013) or sample only sporadically during the 12 to 24 month period (Potier, Meguerditchian, \& Fagard, 2013), or focus only a single manual skill. For example, Sgandurra et al. (2012) examined only prehension (reaching for objects and the adjustment of hand shape in preparation for different grip pattern and force control), whereas Kahrs, Jung, and Lockman (2013) examined the transition of banging into the functional skill of hammering. Unfortunately, in most studies of toddlers, hand-use preferences are ignored or not assessed systematically. Nelson et al. (2013) did report that infant right-hand preference for acquiring objects predicted right-hand preference for RDBM during the 18-24 month age period. This latter result is consistent with a cascading transfer across manual actions. However, the Nelson et al. (2013) study lacked infants with left-hand acquisition preferences and therefore, is not an adequate test of the cascade proposal.

So when and how might hand preferences develop for those without a unimanual preference by 14 months or, even more importantly, for those without a preference for acquisition? The cascade proposal would predict that nearly all of those with a hand preference for acquisition should eventually develop a preference for unimanual manipulation as the acquisition preference is transferred to unimanual manipulation. Of course, parental influences on imitation and hand-use (Harkins \& Uzgiris, 1991; Michel, 1992) and other cultural practices (Michel, 2002) can affect the strength of offspring's hand preference. Although maternal left-handedness is related to offspring left-handedness (Harkins \& Michel, 1988; McKeever, 2000), the vast majority of both potentially left-handed and righthanded offspring are likely to have right-handed mothers. Playful interaction between righthanded mothers and their infants will strengthen offspring right hand use and weaken left hand use (Michel, 1992; Mundale, 1992). Perhaps, this is the reason why there are so few left-handed adults at the extreme left-end of any measure as compared to high frequency of right-handed adults at the extreme right-end. 
Since adult handedness is a continuously distributed variable, especially as measured by performance proficiency (peg-moving, dotting circles), many individuals will exhibit little or no differences between the hands even if they claim self-categorization into one of a few categories (e.g., right, mostly right, equilateral, mostly left, left). We would predict that the majority of the adults with minor differences in proficiency between their hands derive primarily from those with no consistent hand preference for acquiring objects during their first year. Clearly, the early development of handedness deserves more systematic longitudinal investigation.

In conclusion, we observed that the frequency of unimanual manipulations increases during 6 to 14 month age period. Also, a hand preference for unimanual manipulation becomes more distinctive during development, with the infant's hand preference for object acquisition predicting the development of a hand preference for unimanual manipulation. Although the infant's hand preference for object acquisition predicts the development of a hand preference for unimanual manipulation, this hand preference for unimanual manipulation becomes significantly different between those infants with a right versus a left hand preference for acquiring objects only after 10 months. Moreover, the difference seems to be driven by the increasing use of the right hand for those with a right-hand acquisition preference. Although the frequency of unimanual manipulations increased with age, there were sufficient numbers of these actions to identify a hand preference (had there been a preference) for each assessment from 6 to 10 months. Therefore, we propose that the delay in the development of the relation of hand preferences for acquiring objects and hand preferences for unimanually manipulating them is consistent with the prediction that handedness development during infancy involves the transfer of the preference across these two manual skills. Our results support a progressive lateralization notion for the development of handedness in which hand preferences transfer across manual skills in a cascading manner (c.f., Michel, 1983, 1988). We suspect that the transfer is stronger for the use of the right hand because most mothers are right handed and they play with their infant in ways that promote the infant's use of the right hand (Mundell, 1992).

The development of hand preferences for object acquisition and unimanual manipulation during the infant's first year is a complex process that likely establishes the foundation for the development of hand preferences for more sophisticated manual skills (e.g., RDBM, artifact construction, and tool use) during the infant's second year. Artifact construction and tool-use likely involve the coordination of both hands as first manifested in RDBM. The development of hand preferences for object acquisition and unimanual manipulation during the infant's first year likely establishes the foundation for the development of a hand preference for RDBM in children. A hand preference means that those infants manifesting a preference are more likely to manipulate objects and explore their properties differently from those without a preference, which would not only facilitate the development of their manual skills (Kotwica et al., 2008) but also their knowledge of object properties, spatial relations, and logical relations (Langer, 1980). The development of hand preference during infancy also may contribute to the development of other sensorimotor and cognitive functions, including speech control (Michel et al., 2014; Nelson, Campbell, \& Michel, 2014). Thus, infant hand preferences could be an important component in the development of embodied cognitive processes (Smith \& Gasser, 2005). 


\section{Acknowledgments}

We would like to acknowledge the infants and parents that participated in our longitudinal study, and whose patience and help made this research possible. Also, we would like to thank the undergraduate students who helped with the coding of infant data: Richard Jordan, Ron Baron, Jonathan Latta, John Phillip Stevens, and Justin Varholick. These data were used in partial fulfillment for Julie Campbell's Doctoral Dissertation. This research was supported by NSF Grant DLS0718045 awarded to George F. Michel and in part by a pre-doctoral fellowship provided by the National Institute of Child Health and Human Development (T32-HD07376) through the Center for Developmental Science, University of North Carolina at Chapel Hill, to Julie Campbell. Correspondence concerning this article should be addressed to Julie Campbell, Department of Psychology, UNCG, Greensboro, NC 27402 .

\section{References}

Annett M. The distribution of manual asymmetry. British Journal of Psychology. 1972; 63:343-358. [PubMed: 4199291]

Annett, M. Handedness and Brain asymmetry: The Right Shift Theory. Psychology Press; Hove, UK: 2002.

Babik I, Campbell JM, Michel GF. Postural influences on the development of infant lateralized and symmetrical hand-use. Child Development. 2014; 85:294-307. [PubMed: 23638971]

Baldwin, JM. Mental Development in the Child and the Race: Methods and Processes. The Macmillan Co.; New York: 1894.

Briggs GG, Nebes RD. Patterns of hand preference in a student population. Cortex. 1975; 11(3):230238. [PubMed: 1204363]

Cohen AI. Hand preference and developmental status of infants. Journal of Genetic Psychology. 1966; 108:337-345. [PubMed: 5961989]

Ferre CL, Babik I, Michel GF. Development of infant prehension handedness: A longitudinal analysis during the 6- to 14-month age period. Infant Behavior and Development. 2010; 33:492-502. [PubMed: 20619463]

Harkins DA, Uzgiris IC. Hand-use matching between mothers and infants during the first year. Infant Behavior and Development. 1991; 14:289-298.

Hildreth G. The development and training of hand preferences. Journal of Genetic Psychology. 1949; 75:197-220. [PubMed: 15409766]

Hinojosa T, Sheu C-F, Michel GF. Infant hand-use preferences for grasping objects contributes to the development of a hand-use preference for manipulating objects. Developmental Psychobiology. 2003; 43:328-334. doi:10.1002/dev.10142. [PubMed: 15027416]

Jones BL, Nagin DS, Roeder K. A SAS Procedure Based on Mixture Models for Estimating Developmental Trajectories. Sociological Methods and Research. 2001; 29:374-393.

Kahrs BA, Jung WP, Lockman JJ. Motor origins of tool use. Child Development. 2013; 84(3):810816. [PubMed: 23106197]

Kass RE, Raftery AE. Bayes factors. Journal of the American Statistical Association. 1995; 90:773795.

Kass RE, Wasserman L. A reference Bayesian test for nested hypotheses and its relationship to the Schwarz criterion. Journal of the American Statistical Association. 1995; 90:928-934.

Kimmerle M, Ferre CL, Kotwica KA, Michel GF. Development of role-differentiated bimanual manipulation during the infant's first year. Developmental Psychobiology. 2010; 52:168-180. doi: 10.1002/dev.20428. [PubMed: 20127887]

Kimmerle M, Mick LA, Michel GF. Bimanual role-differentiated toy play during infancy. Infant Behavior and Development. 1995; 18:299-307.

Kotwica KA, Ferre CL, Michel GF. Relation of stable hand-use preferences to the development of skill for managing multiple objects from 7 to 13 months of age. Developmental Psychobiology. 2008; 50:519-529. [PubMed: 18551469]

Langer, J. The Origins of Logic: Six to Twelve Months. Academic Press; New York: 1980.

McKeever WF. A new family handedness sample with findings consistent with X-linked transmission. British Journal of Psychology. 2000; 91:21-39. [PubMed: 10717769] 
Michel, GF. Development of hand-use preference during infancy.. In: Young, G.; Segalowitz, S.; Corter, C.; Trehub, S., editors. Manual Specialization and the Developing Brain. Academic Press; New York: 1983. p. 33-70.

Michel, GF. A neuropsychological perspective on infant sensorimotor development.. In: RoveeCollier, C.; Lipsitt, LP., editors. Advances in Infancy Research. Vol. 5. Ablex; Norwood, NJ: 1988. p. 1-38.

Michel GF. Maternal influences on infant hand-use during play with toys. Behavior Genetics. 1992; 22:163-176. [PubMed: 1596256]

Michel GF. A lateral bias in the neuropsychological functioning of human infants. Developmental Neuropsychology. 1998; 14:445-469.

Michel, GF. Development of infant handedness.. In: Lewkowicz, DJ.; Lickliter, R., editors. Conceptions of Development: Lessons from the Laboratory. Psychology Press; New York: 2002. p. 165-186.

Michel GF, Babik I, Sheu C-F, Campbell JM. Latent classes in the developmental trajectories of infant handedness. Developmental Psychology. 2014; 50:349-359. [PubMed: 23772820]

Michel, GF.; Nelson, EL.; Babik, I.; Campbell, JM.; Marcinowski, EC. Multiple trajectories in the developmental psychobiology of human handedness.. In: Lerner, RM.; Benson, JB., editors. Embodiment and Epigenesis: Theoretical and Methodological Issues in Understanding the Role of Biology within the Relational Developmental System (Part B: Ontogenetic Dimensions. Elsevier Inc.: Academic Press; 2013. p. 227-260.

Mundale, CJ. Influences of maternal handedness and behavior on infant hand use preferences (Unpublished master's thesis). DePaul University; Chicago, IL.: 1992.

Nagin, D. Group-Based Modeling of Development. Harvard Univ Press; Cambridge, MA: 2005.

Nelson EL, Campbell JM, Michel GF. Unimanual to bimanual: Tracking the development of handedness from 6 to 24 months. Infant Behavior and Development. 2013; 36:181-188. [PubMed: 23454419]

Nelson EL, Campbell JM, Michel GF. Early handedness in infancy predicts language ability in toddlers. Developmental Psychology. 2014; 50:809-814. doi: 10.1037/a0033803. [PubMed: 23855258]

Piaget, J. The Origins of Intelligence in Children. International Universities Press; New York: 1952.

Potier C, Meguerditchian A, Fagard J. Handedness for bimanual coordinated actions in infants as a function of grip morphology. Laterality. 2013; 18:576-593. [PubMed: 23231501]

Ramsay DS. Onset of unimanual handedness in infants. Infant Behavior and Development. 1980; 3:377-385.

Raudenbush, S.; Bryk, A.; Cheong, YF.; Congdon, R.; du Toit, M. HLM 6: Hierarchical Linear and Nonlinear Modeling. Scientific Software International, Inc.; Lincolnwood, IL: 2004.

Sgandurra G, Cecchi F, Serio SM, Del Maestro M, Laschi C, Dario P, Cioni G. Longitudinal study of unimanual actions and grasping forces during infancy. Infant Behavior and Development. 2012; 35:205-214. [PubMed: 22297129]

Siegel, S. Nonparametric Statistics for the Behavioral Sciences. McGraw-Hill; 1956.

Smith L, Gasser M. The development of embodied cognition: Six lessons from babies. Artificial Life. 2005; 11:13-29. [PubMed: 15811218]

Steenhuis RE, Bryden MP. Different dimensions of hand preference that relate to skilled and unskilled activities. Cortex. 1989; 25:289-304. [PubMed: 2758854]

Todor JI, Doane T. Handedness classification: preference versus proficiency. Perceptual and Motor Skills. 1977; 45:1041-1042. [PubMed: 604877]

Touwen, B. Lavenham Press; Suffolk, UK: 1976. Neurological development in infancy..

Uzgiris, IC.; Hunt, JM. Assessment in infancy. University of Illinois Press; Chicago: 1975. 
- Development of hand preference for unimanual manipulation was assessed during infancy

- Hand-use preferences for unimanual manipulation appear only after 10 months of age

- Hand-use preferences for acquiring objects are present from 6 months on

- Hand-use preferences for acquiring objects predicted preferences for manipulation

- Many infants do not exhibit a preference for unimanual manipulation even by 14 months 


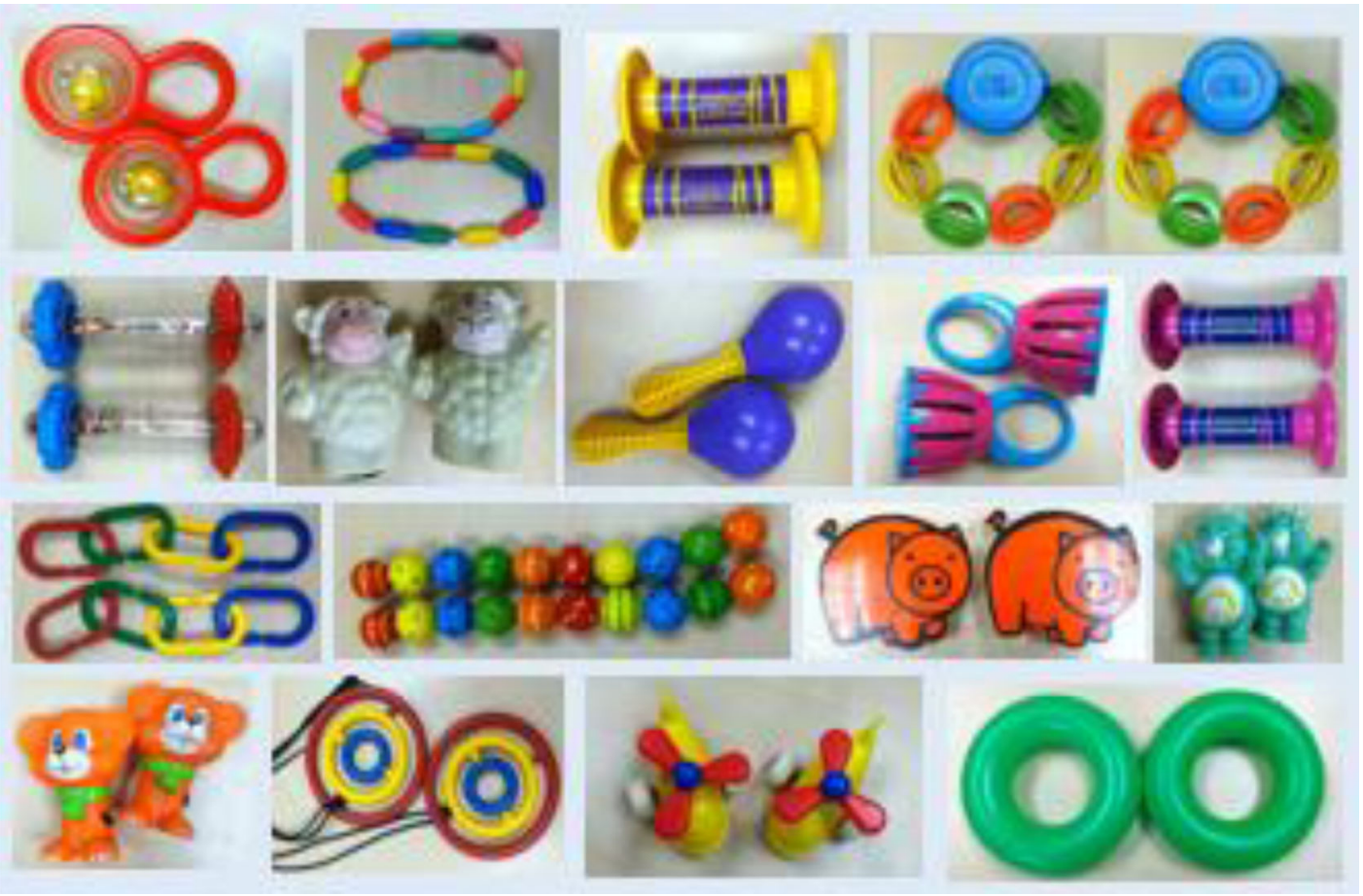

Figure 1.

The 17 pairs of items used in the unimanual task. 


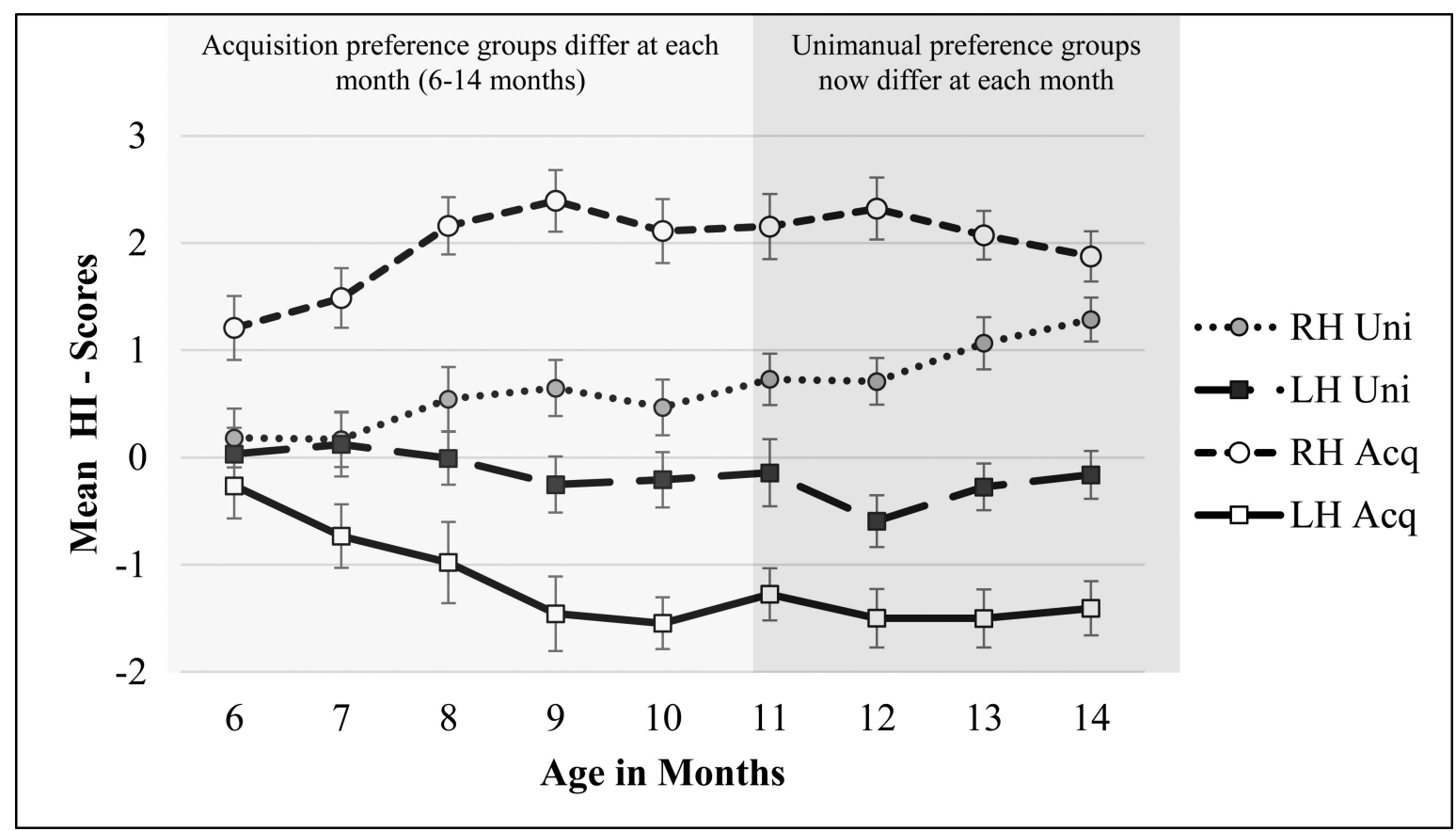

Figure 2.

Mean (and standard errors) HI scores for acquiring objects and for unimanual manipulation for infants classified by their latent class trajectory analysis as having right and left hand preferences for acquiring objects. 


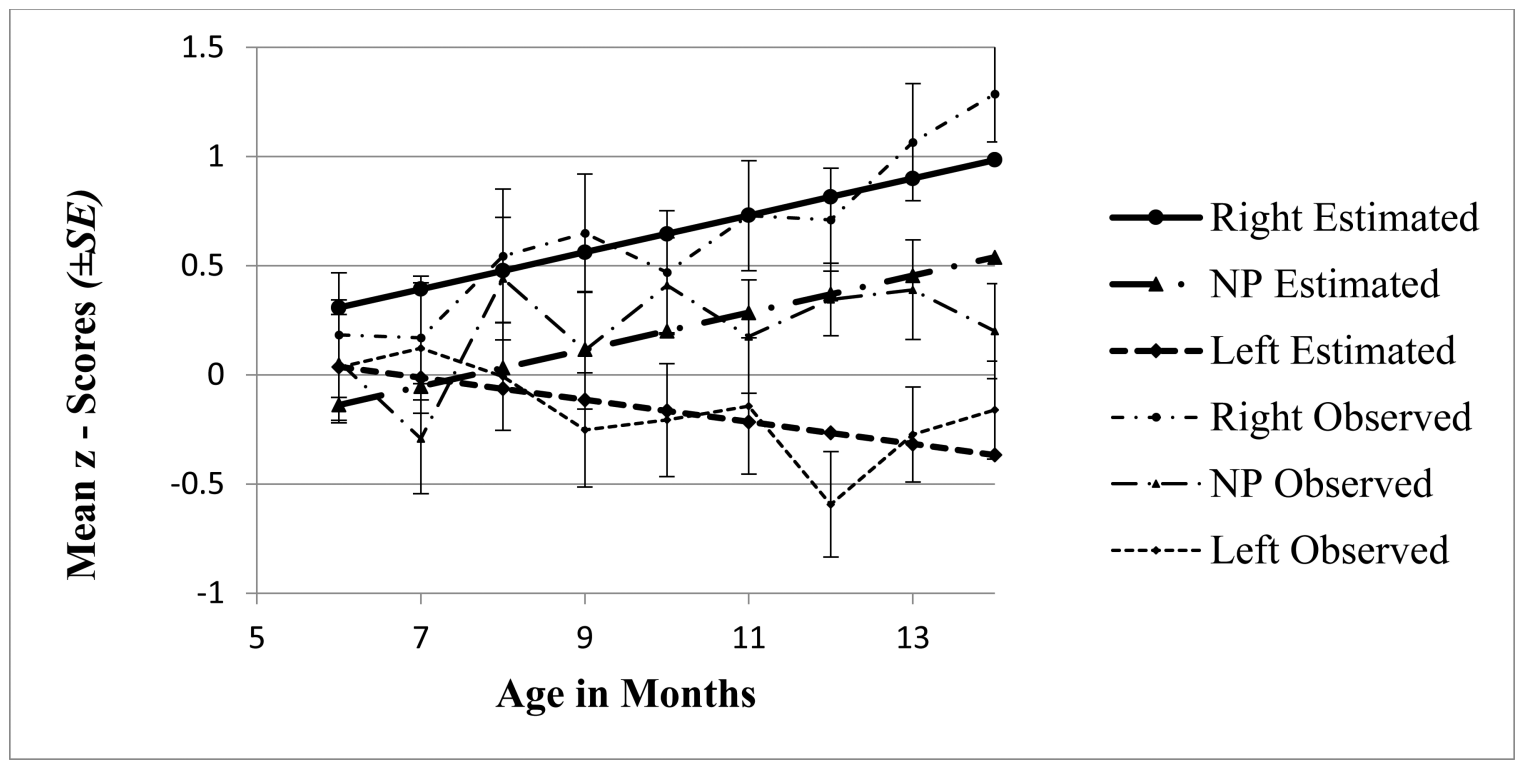

Figure 3.

Estimated and observed trajectories of change in hand preference for unimanual manipulations, NP = no preference. 


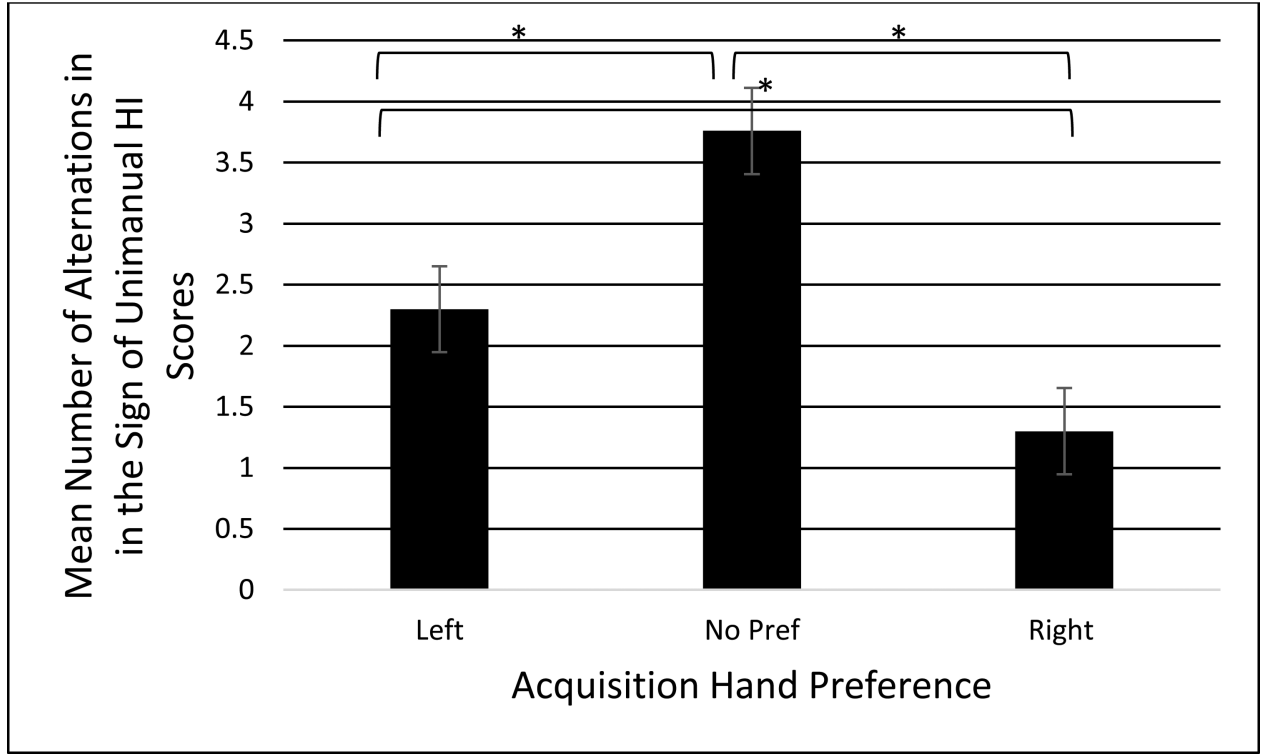

Figure 4.

Mean number of alternations of the signs for HI scores for unimanual hand preference according to each acquisition hand preference group. The no preference group exhibits the most shifts indicating the least consistency in their unimanual preference. 


\section{Table 1}

Estimated Fixed and Random Effects for Unimanual Manipulations According to Hand Preference for Acquiring Objects.

\begin{tabular}{|c|c|c|c|}
\hline Level 1 Effects & Level 2 Effects & Parameters & Manipulation \\
\hline & \multicolumn{3}{|c|}{ Fixed Effects } \\
\hline \multirow[t]{3}{*}{ Initial status, $\pi_{0 \mathrm{i}}$} & Intercept & $\beta_{00}$ & -0.200 \\
\hline & Left & $\beta_{01}$ & 0.541 \\
\hline & NP & $\beta_{02}$ & $-0.446^{* *}$ \\
\hline \multirow[t]{2}{*}{ AGE, $\pi_{1 \mathrm{i}}$} & Intercept & $\beta_{10}$ & $0.085^{* * *}$ \\
\hline & Left & $\beta_{11}$ & $-0.135^{* * *}$ \\
\hline \multirow[t]{3}{*}{$\mathrm{AGE}^{2}, \pi_{2 \mathrm{i}}$} & Intercept & $\beta_{20}$ & \\
\hline & Left & $\beta_{21}$ & \\
\hline & \multicolumn{3}{|c|}{ Random Effects } \\
\hline Level 1: & Within-person, $\varepsilon_{\mathrm{ij}}$ & $\sigma \varepsilon^{2}$ & 1.733 \\
\hline Level 2: & Intercept, $\delta_{0 \mathrm{i}}$ & $\sigma_{0}^{2}$ & $0.209^{* * *}$ \\
\hline \multicolumn{4}{|l|}{ Note. } \\
\hline \multicolumn{4}{|l|}{$* p<.05$} \\
\hline \multicolumn{4}{|l|}{$\mathrm{NP}=$ no preference } \\
\hline$* * * \leq .01$ & & & \\
\hline$* * * * 0.001$. & & & \\
\hline
\end{tabular}




\section{Table 2}

Number of Infants in Each Hand Preference Category According to their Acquisition Hand Preference (from the Latent Class Analysis of Developmental Trajectories across the 9 Months) and Unimanual Hand Preference (from the HI-score Classification of Hand-use for each Month).

\begin{tabular}{ccccccccccc}
\hline & \multicolumn{9}{c}{ Hand Preference for Acquiring Objects } \\
\cline { 2 - 10 } Infant Age in Months & \multicolumn{3}{c}{ Right } & \multicolumn{5}{c}{ Left } & \multicolumn{1}{c}{ No Preference (NP) } \\
\cline { 2 - 10 } & \multicolumn{4}{c}{ Hand Preference for Unimanual Manipulation } \\
\cline { 2 - 11 } & Right & NP & Left & Right & NP & Left & Right & NP & Left \\
\hline 6 & 4 & 22 & 4 & 5 & 23 & 2 & 4 & 21 & 3 \\
7 & 5 & 21 & 4 & 5 & 20 & 4 & 4 & 22 & 4 \\
8 & 8 & 18 & 3 & 2 & 24 & 3 & 7 & 20 & 3 \\
9 & 6 & 21 & 3 & 3 & 23 & 4 & 6 & 18 & 5 \\
10 & 10 & 17 & 2 & 2 & 24 & 3 & 5 & 23 & 2 \\
11 & 5 & 24 & 1 & 5 & 20 & 5 & 4 & 24 & 2 \\
12 & 8 & 22 & 0 & 1 & 22 & 7 & 3 & 27 & 0 \\
13 & 10 & 18 & 2 & 0 & 26 & 4 & 4 & 25 & 1 \\
14 & 14 & 16 & 0 & 2 & 23 & 5 & 0 & 29 & 1 \\
\hline
\end{tabular}

Infant Behav Dev. Author manuscript; available in PMC 2016 May 01. 